\title{
Impact of OLTC equipped transformer operation on PV installation in urban distribution network
}

\author{
N. Srećković, N. Lukač and G. Štumberger \\ University of Maribor \\ Faculty of Electrical Engineering and Computer Science \\ Koroška cesta 46, 2000 Maribor, Slovenija \\ Phone number:+386 220 7056, e-mail: nevena.sreckovic@um.si, niko.lukac@um.si, gorazd.stumberger@um.si
}

\begin{abstract}
Presented paper addresses the problem of proper large-scale integration of photovoltaic (PV) systems in urban area distribution network (DN). A methodology for minimization of annual energy losses in DN by optimal placement of PV systems, which simultaneously considers rooftop PV potential and timedependent network operation, is presented. Optimal reactive power generation of PV systems and optimal setting of on-load tap changer (OLTC) equipped transformer in secondary substation are determined as well. Furthermore, proposed methodology enables evaluation of the impact of OLTC equipped transformer on the share of PV installation that urban DN can accommodate. Proposed methodology is based on the optimization tool called differential evolution, with the objective function set to minimize network's annual energy losses, while preventing the voltage violations and thermal overloading of lines. Results of the case study performed on a real urban low voltage distribution network, with consideration of different scenarios of OLTC operation are presented. The results indicate that simultaneous consideration of chosen variables yields greater benefits to DN operation in terms of reduction of annual energy losses and greater PV penetration.
\end{abstract}

\section{Key words}

PV integration, loss minimization, $\mathrm{PV}$ potential, reactive power control, OLTC.

\section{Introduction}

Urban area distribution networks (DN) are expected to accommodate rapidly increasing share of distributed generation (DG) units, especially photovoltaic systems (PV), due to political directives and incentives promoting their integration [1]. This phenomenon, however, could cause problems with voltage rise beyond the statuary limits. Furthermore, with European Commission's Energy Efficiency Winter Package 2016 [2], active participation of different elements of DN and novel algorithms of DN design, operation, control and optimization are being promoted. Therefore, with proper operation of active elements in the network, such as voltage control devices, reactive power control, real time network reconfiguration, demand side management etc., low voltage (LV) networks could accommodate greater shares of PV systems, than previously possible [3]. In addition to ensuring the proper voltage profiles, optimization of distribution network operation, in terms of minimization of energy losses could also be achieved.

This paper presents the methodology for assessing the benefits of optimal installation of PV systems in real, urban, low voltage distribution networks, with simultaneous consideration of PV potential of rooftop surfaces and time-dependent network operation. Optimal reactive power generation of $\mathrm{PV}$ systems and optimal setting of on-load tap changer (OLTC) equipped transformer in secondary substation, supplying the network, are determined as well. Previous work of the authors proposed a methodology for determining rooftop surfaces, suitable for installation of PV systems, based on LiDAR (Light Detection And Ranging) data, pyranometer measurements, and time-dependent network operation [4], and employed the PV potential assessment method, presented in [5]. The work showed that most optimal surfaces, with respect to PV potential, are not necessarily the best from the network operation point of view, and that detailed consideration of time-dependent network operation should be taken into account as well. In this work, not only the time-dependent power generation and consumption are considered, but also the generation of reactive power of PV systems, as well as optimal setting of OLTC. Evaluation of enhancement of PV penetration in LV networks due to optimal OLTC operation can be found in literature [6], [7], suggesting that OLTC equipped secondary substations are yet to be utilized with modernization of the LV networks.

Presented paper is structured as follows. Second section explains in detail the proposed methodology for minimization of annual electric energy losses by simultaneous consideration of PV potential, timedependent power generation and consumption, reactive power generation and OLTC settings. Third section presents the real urban low voltage network and four different OLTC operation scenarios, chosen for the case 
study of the proposed methodology. Fourth section presents the results of the annual reduction of energy losses and selection of optimal rooftop surfaces for installation of PV systems for considered scenarios of OLTC operation, thus allowing the evaluation of the impact of OLTC operation on PV installations in urban DN. Final section concludes the paper and gives the final remarks.

\section{Proposed methodology}

The flowchart of the proposed procedure for minimization of the annual electric energy losses, by installation of PV systems on locations, optimal from the standpoint of both network operation and PV potential, with simultaneous consideration of reactive power generation and OLTC setting is presented in Fig. 1. Algorithm firstly evaluates the annual network losses of the original network. Calculation of optimal additional active and reactive power generation and OLTC tap operation, yielding minimum annual energy losses follows. In the final step, rooftop surfaces that ensure the required active power generation are chosen for installation of PV systems. Detailed description of individual steps in the given flowchart is given hereinafter.

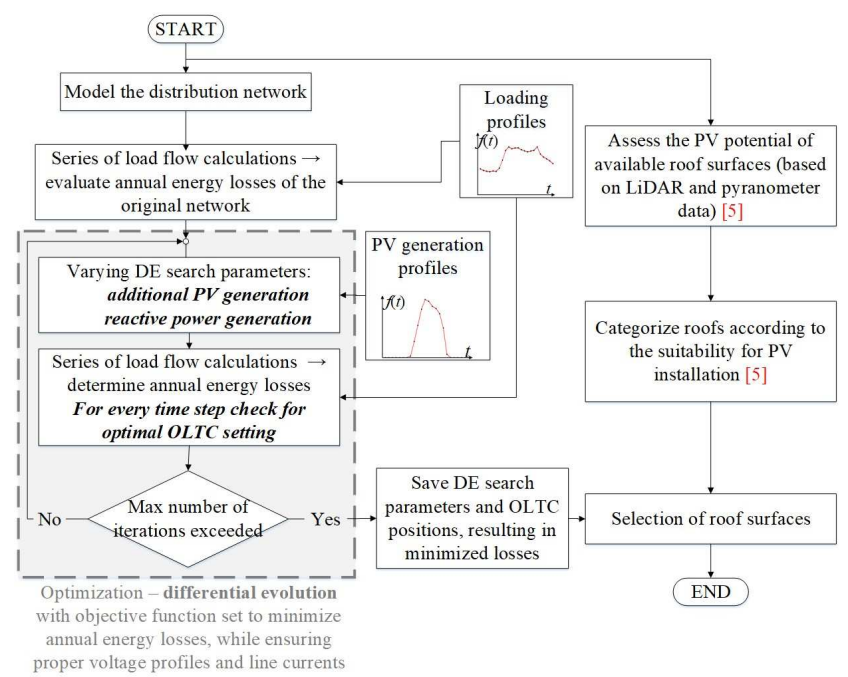

Fig. 1: Flowchart of the proposed procedure for minimization of annual energy losses.

Firstly, annual electric energy losses are determined for the original network operation, without consideration of the OLTC. Evaluation of losses in a specific time point is performed using the load flow calculation, suitable for radial distribution network (backward-forward sweep method [8]). Hourly loading profiles for the points of common coupling (PCC) in the discussed network, for average day of every month, are created based on longterm measurements on the transformer substation supplying the network. By performing a series of load flow calculations for every hour of average day in a month, calculation of power losses and thus annual energy losses is possible.

Assessment of PV potential of rooftop surfaces is performed based on areal LiDAR scanning data, and by long-term solar irradiance measurements made with pyranometer. Nonlinear efficiency characteristics of PV modules with solar inverters, their inclination and shadowing from surrounding territory and vegetation are considered as well. Based on these data, hourly profiles of power generated by monocrystalline PV systems that could be placed on considered rooftop surfaces are determined. Evaluated rooftop surfaces are categorized by suitability for PV installations, considering their average solar irradiance, thus distinguishing between more to less preferable surfaces for placement of PV systems.

Following step in the proposed methodology is the optimisation part, with optimal solution defined as the one yielding the minimum annual energy losses. Objective function, satisfying such definition is given as the quotient of annual energy losses of the DN with added PV generation $W_{\text {loss_addPV }}$, and annual energy losses of the original network without the consideration of the additional PV generation $W_{\text {loss_origDN }}$ (1). Penalties $p$ ensure that the voltage profiles and thermal loading of lines are kept within the required limits.

$q_{\text {fun }}=\min \frac{W_{\text {loss_addPV }}}{W_{\text {loss_origDN }}}+p$

Optimization tool chosen for the proposed methodology is differential evolution (DE) [9]. The goal of DE is to find such set of search parameters $\boldsymbol{x}_{\mathrm{p}}$, which will satisfy given objective function (1). It is a metaheuristic procedure that mimics the process of evolution in nature, by considering mutation, crossover and selection of search parameters, thus ensuring the exploration and exploitation of search space and reducing the chance of getting stuck in a local optimum. In this study, number of DE search parameters $n$ corresponds to the number of PCC in the LV network, considered for installation of PV systems onto their rooftop. For each PCC, a share of possible active power generation from the power available on the adjacent rooftop surfaces is determined. If the optimum additional power generation for a PCC $i$ is obtained by taking into account all adjacent roofs, the value of search parameter $x_{\mathrm{p}, \mathrm{i}}$ is 1 (i.e. $100 \%$ ). If only part of the available power is required, search parameter gives the required share. Additional search parameter $x_{\mathrm{p}, \mathrm{n}+1}$ is introduced in order to determine the optimal reactive power generation, which is defined as a relative share of possible active power generation from the considered PV systems. It is represented with a single parameter, meaning that all PV systems will operate with the same power factor, which will be constant throughout the year. Operation of OLTC will, depending from simulation scenario, be constant throughout the year, or its monthly or hourly operation will be enabled, allowing the assessment of impact of OLTC equipped transformer operation on PV installation.

After DE determines the optimal additional PV generation per each considered PCC in the network, their reactive power generation and optimal OLTC operation, the final step of the presented methodology remains. Set of rooftop surfaces capable of generating power determined in DE must be selected from the set of all 
considered surfaces. The selection is performed in a way, that rooftop surfaces, more suitable for the installation from solar potential point of view are selected first, followed by those of lower ratings. This way selection of minimum number of rooftops, guaranteeing demanded generation, is ensured.

\section{Case study}

Real urban LV network chosen for demonstration of the proposed methodology is presented in Fig. 2. Polygons in the figure represent rooftop surfaces, whose solar irradiance and hourly values of PV potential have been determined in previous work [5]. Surfaces are categorized into four suitability categories, according to the solar irradiance they are exposed to. Secondary transformer substation is supplying $24 \mathrm{PCC}$, with 69 rooftop surfaces considered, adjacent to 22 PCC of the network. For remaining two $\mathrm{PCC}$, data regarding solar irradiance on adjacent rooftops was unavailable. This paper assumes that the transformer in secondary substation, supplying the network, is equipped with OLTC, with total voltage range of $\pm 12 \%, 9$ tap positions and $3 \%$ voltage range per step (i.e. $\pm 4 \times 3 \%$ ).

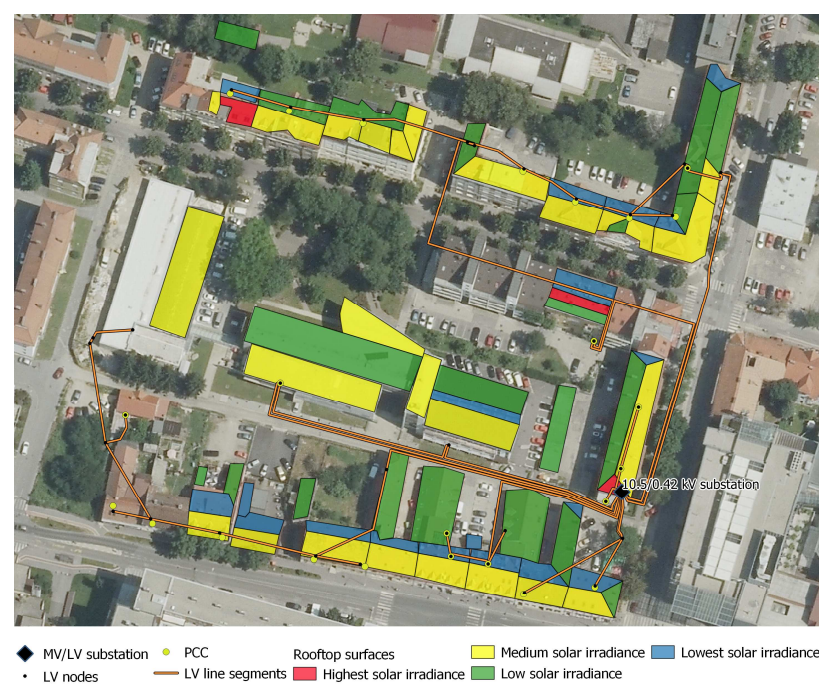

Fig. 2: Discussed real urban LV distribution network.

Following four scenarios of OLTC operation will be considered and results of preformed simulations will be presented in the following section.

\section{- Scenario 1: no OLTC}

In this scenario, optimal active and reactive power generation are determined. Voltage of the slack bus (LV busbar of the transformer) is set to correspond to neutral tap position of OLTC (as if it were not there), which is 0 .

- Scenario 2: optimal annual OLTC tap position In this scenario, optimal active and reactive power generation are determined for different OLTC tap positions that are considered constant throughout the year.

\section{- Scenario 3: optimal monthly OLTC tap position}

In this scenario, optimal active and reactive power generation are determined, for different OLTC tap positions that are considered constant throughout each month (i.e. optimal tap position is determined for each month).

\section{- Scenario 4: optimal hourly OLTC tap position}

In this scenario, optimal active and reactive power generation are determined, with hourly change of tap position allowed (i.e. optimal tap position is determined for every hour of average day in a month).

In each scenario, after determining the optimal share of PV generation per each PCC, rooftop surfaces, yielding such generation are selected. Rated power $(\mathrm{kWp})$ of required monocrystalline PV systems is determined as well.

\section{Results}

Obtained results for the case study of four OLTC operation scenarios on the real urban LV distribution network, presented in the previous section, is given hereinafter.

Table I gives the reduction of annual energy losses (with respect to the original $\mathrm{DN}$, without the additional $\mathrm{PV}$ systems), and rated power of the PV systems required for obtaining such reduction, for chosen operation scenarios. Number of selected rooftop surfaces, yielding determined rated power, from 69 rooftop surfaces present in the network model is given as well.

Table I: Simulation results for four OLTC operation scenarios.

\begin{tabular}{|c|c|c|c|c|c|}
\cline { 3 - 6 } \multicolumn{2}{c|}{} & Scenario 1 & Scenario 2 & Scenario 3 & Scenario 4 \\
\hline \multicolumn{2}{|c|}{ Loss reduction (\%) } & 27.7 & 37.6 & 39.3 & 39.9 \\
\hline \multicolumn{2}{|c|}{ rated power of PV systems (kWp) } & 268.3 & 204.3 & 264.7 & 264.1 \\
\hline \multirow{2}{*}{$\begin{array}{c}\text { number of chosen } \\
\text { rooftop surfaces }\end{array}$} & whole surface & 32 & 14 & 31 & 31 \\
\cline { 2 - 6 } & partial surface & 11 & 19 & 11 & 11 \\
\hline
\end{tabular}

Rooftop surfaces, chosen as the best locations for installation of PV systems, by considering both PV potential and time-dependent network operation are presented in Fig. 3 to Fig. 6. Percentages represent required parts of individual rooftop surface.

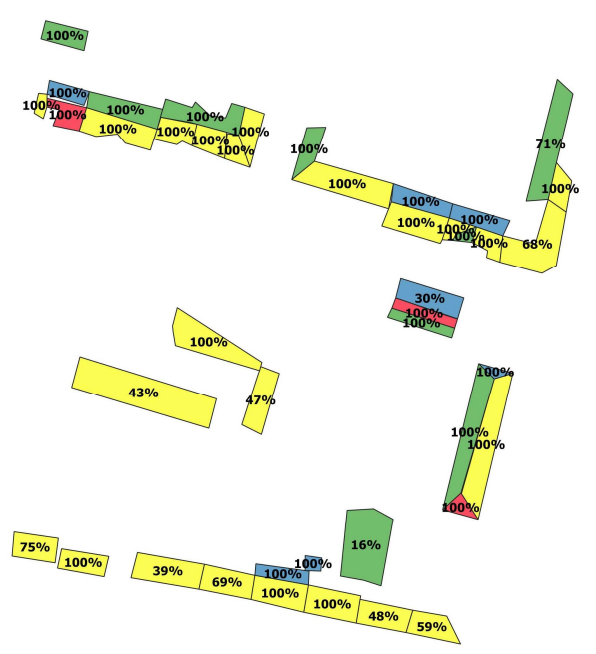

Fig. 3: Selected rooftop surfaces - scenario 1 . 


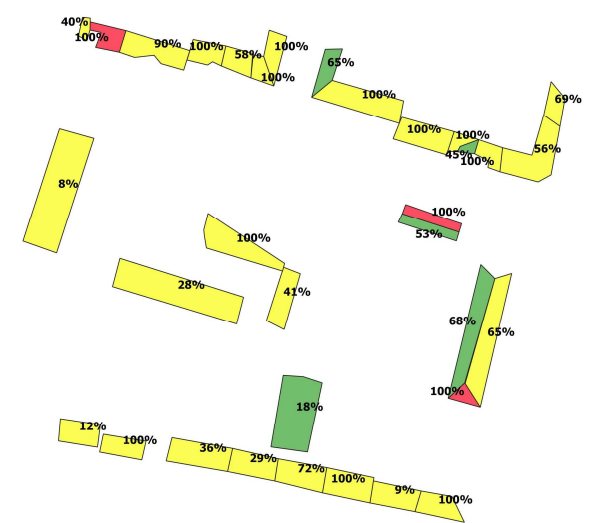

Fig. 4: Selected rooftop surfaces - scenario 2.

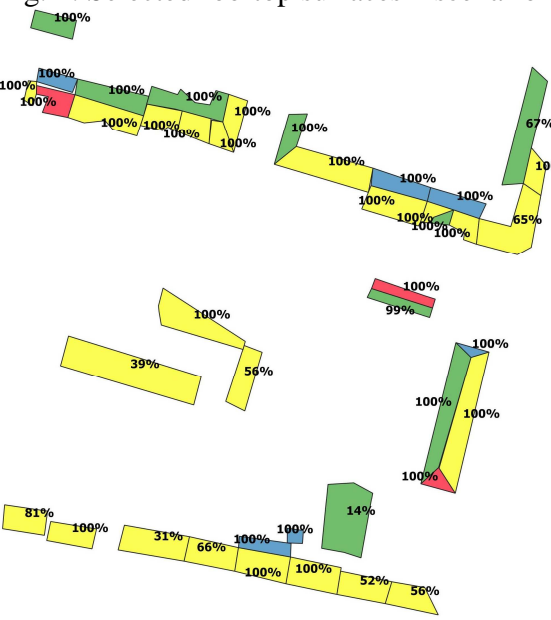

Fig. 5: Selected rooftop surfaces - scenario 3.

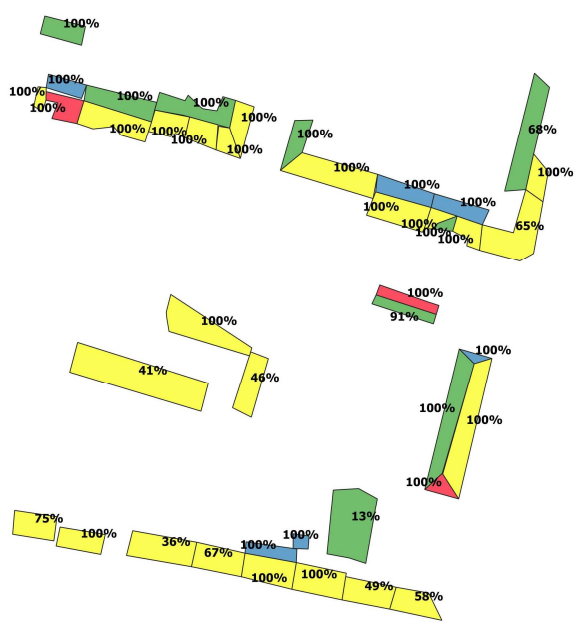

Fig. 6: Selected rooftop surfaces - scenario 4.

Fig. 7 presents the values of search parameters, determined by DE for four OLTC operation scenarios. First 22 values (PCC 1 - PCC 22), represent percentage shares of available power generation for each PCC, yielding minimum annual energy losses. Last, $23^{\text {rd }}$ search parameter gives information regarding optimal generation of reactive power, given as a share of determined active power.

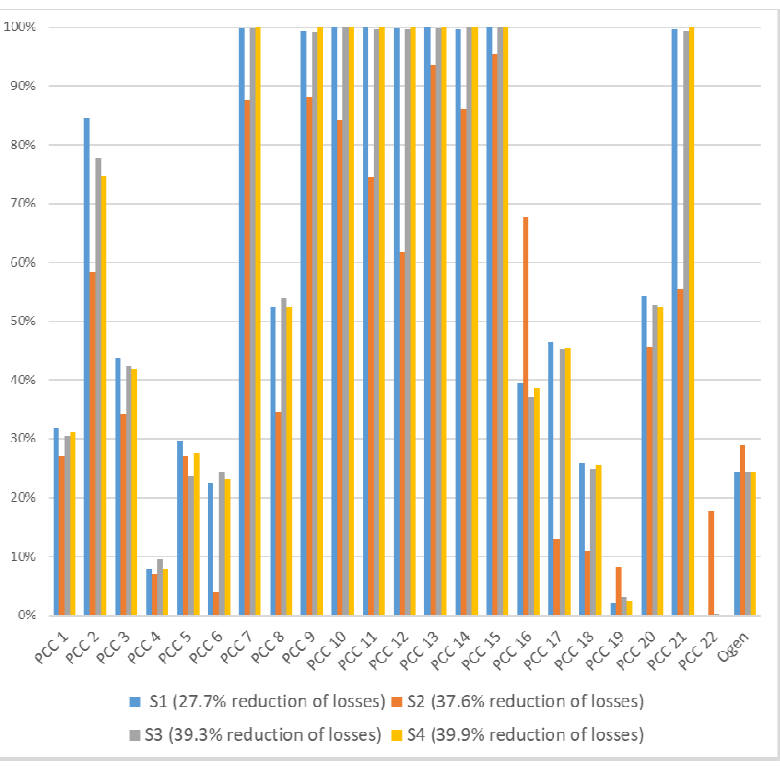

Fig. 7: Optimal additional PV generation per PCC, given as share of available generation with respect to PV potential.

From the results presented in Table I and Fig. 3 to Fig. 7 follows that operation scenario of OLTC equipped transformer has significant impact on both PV installation and energy losses in the DN.

Without the consideration of OLTC (scenario 1), optimal placement of PV systems of $268.3 \mathrm{kWp}$ rated power yields in $27.7 \%$ reduction of annual energy losses. In the second scenario, additional $10 \%$ reduction of losses can be achieved, by setting tap position to +3 . However, by increasing the voltage profile (and thus reducing the power losses), distribution network can accommodate smaller amounts of PV systems, without violating voltage limitations.

If monthly change of OLTC tap position is allowed (scenario 3), 12\% more reduction of annual energy losses is obtained, with almost the same rated power and selection of rooftop surfaces as in scenario 1 . Furthermore, by allowing the change of tap position once per month from ideal position +3 , obtained in scenario 2 , $60 \mathrm{kWp}$ more PV systems can be accommodated and $2 \%$ more reduction of energy losses achieved. Optimal monthly tap positions are presented in Table II. The results show that only two changes of tap position per year are required, between "summer" (May to August) and "winter" months (September to April). During months with higher power generation from PV systems, it is optimal to reduce tap position from +3 to +2 , thus somewhat increasing the losses, but allowing the greater accommodation of PV systems. With approach of colder months, tap position is set back to value +3 .

Table II: Optimal monthly OLTC tap positions.

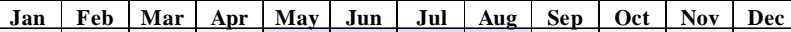

\begin{tabular}{c|c|c|c|c|c|c|c|c|c|c|c|} 
Jan & Feb & Mar & Apr & May & Jun & Jul & Aug & Sep & Oct & Nov & Dec \\
\hline+3 & +3 & +3 & +3 & +2 & +2 & +2 & +2 & +3 & +3 & +3 & +3 \\
\hline
\end{tabular}

In scenario 4, optimal tap position was determined for every hour of every day in a month. Results obtained are almost identical to the scenario 3, with the difference in additional $0.6 \%$ of reduction of energy losses, with same 
rooftop surfaces selected as most optimal for placement of PV systems.

Table III: Optimal OLTC tap positions for every hour of average day in a month.

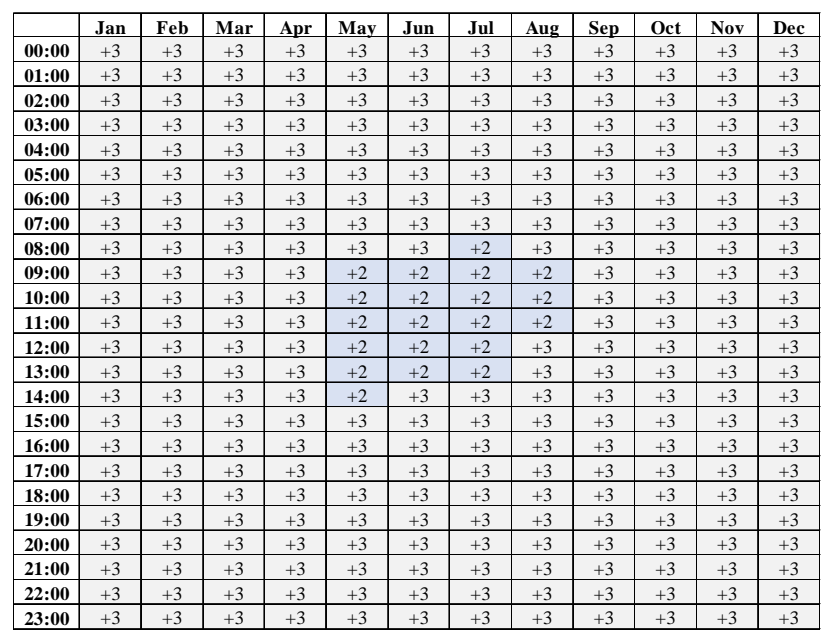

Table III gives results of optimal OLTC tap positions for every hour of average day in a month. The results of tap operation are similar to the ones obtained in scenario 3, with change of tap position being required only during the warmer months from April to August. Furthermore, by allowing the OLTC operation during the day as well, it is seen that during these months only two tap operations per day are required. In the morning, when PV systems start generating power, tap position is lowered from +3 to +2 . Second operation is required in the early afternoon, when tap position can be set back to value +3 .

\section{Conclusion}

Presented paper introduced the methodology for placement of PV systems, on locations, optimal from the standpoint of both time-dependent network operation and PV potential, with simultaneous consideration of reactive power generation and OLTC operation. Optimal solution was considered to be the one, resulting in minimization of annual losses of electric energy, without the violation of voltage profiles and current limitations. Presented method was used to evaluate the impact of OLTC equipped transformer operation on a large-scale PV installation in urban distribution network. Case study on a real, urban low voltage distribution network was performed for four OLTC operation scenarios. As it has been shown in our previous work, rooftop surfaces, highly suitable for PV installation regarding the PV potential, are not necessarily optimal from the DN operation standpoint, as they may cause the reverse power flows, increase of losses or violations of voltage profiles. Therefore, it is important to include different parameters of network operation, when sitting and sizing new generation units, and this paper included the consideration of OLTC equipped transformer.

Increasing the voltage profile in the network by setting the higher OLTC tap position, fixed throughout the year, can lead to additional $10 \%$ reduction of power losses, with respect to neutral OLTC tap position. However, it also results in a significant reduction of rated power of PV system that network can accommodate (from $268.3 \mathrm{kWp}$ to $204.1 \mathrm{kWp})$. Almost identical reduction of annual power losses can be achieved by allowing monthly or hourly change of tap position The results of simulation scenarios 3 and 4 indicate that for the discussed distribution network, wear and tear due to increased OLTC operation would not be a problem, as tap operation would be required only during the summer months, twice per day (in the morning and in the afternoon). 


\section{Acknowledgement}

This work is supported in part by the Slovenian Research Agency under research contracts: L2-5489, P2-0115, and J2-6764.

\section{References}

[1] E. Parlament, "Directive 2009/28/EC of the European Parlament and of the Council of 23 April 2009 on the promotion of the use of energy from renewable sources," 2009.

[2] E. Commission, "Energy Efficiency Directive Winter package 2016," Brussels, 2016.

[3] M. M. Haque and P. Wolfs, "A review of high PV penetrations in LV distribution networks: Present status, impacts and mitigation measures," Renew. Sustain. Energy Rev., vol. 62, pp. 1195-1208, Sep. 2016.

[4] N. Srećković, N. Lukač, B. Žalik, and G. Štumberger, "Determining roof surfaces suitable for the installation of PV (photovoltaic) systems, based on LiDAR (Light Detection And Ranging) data, pyranometer measurements, and distribution network configuration," Energy, vol. 96, pp. 404-414, Feb. 2016.

[5] N. Lukač, S. Seme, D. Žlaus, G. Štumberger, and B. Žalik, "Buildings roofs photovoltaic potential assessment based on LiDAR (Light Detection And Ranging) data," Energy, vol. 66, pp. 598-609, Mar. 2014.

[6] T. Aziz and N. Ketjoy, "Enhancing PV Penetration in LV Networks Using Reactive Power Control and On Load Tap Changer With Existing Transformers," IEEE Access, vol. 6, pp. 2683-2691, 2018.

[7] A. Navarro-Espinosa and L. F. Ochoa, "Increasing the PV hosting capacity of LV networks: OLTC-fitted transformers vs. reinforcements," in 2015 IEEE Power \& Energy Society Innovative Smart Grid Technologies Conference (ISGT), 2015, pp. 1-5.

[8] D. Thukaram, H. M. Wijekoon Banda, and J. Jerome, "A robust three phase power flow algorithm for radial distribution systems," Electr. Power Syst. Res., vol. 50, no. 3, pp. 227-236, 1999.

[9] R. Storn and K. Price, "Differential Evolution - A Simple and Efficient Heuristic for global Optimization over Continuous Spaces," J. Glob. Optim., vol. 11, no. 4, pp. 341-359, 1997. 